\title{
Conhecimento de manipuladores de carne sobre o complexo teniose-cisticercose e Boas Práticas de Manipulação de alimentos em Jaboticabal, São Paulo, Brasil**
}

\section{Meat handlers' knowledge of taeniosis-cysticercosis complex and good manufacturing practices in Jaboticabal, São Paulo State, Brazil}

\author{
Bruna Ferreira Izola, ${ }^{* *}$ Gabriel Augusto Marques Rossi, ${ }^{* *}$ Mirelle Andréa de Carvalho Picinato, ${ }^{* *}$ \\ Estevam Guilherme Lux Hoppe, ${ }^{* *}$ Ana Maria Centola Vidal Martins, ${ }^{* *}$ Karina Paes Bürger ${ }^{* *}$
}

\begin{abstract}
Resumo
O estudo objetivou avaliar o conhecimento de manipuladores de carne do município de Jaboticabal, estado de São Paulo, sobre o complexo teniose-cisticercose e sobre as Boas Práticas de Manipulação dos alimentos. Participaram do estudo 25 estabelecimentos, dos quais nove de pequeno porte, dez de médio porte e seis de grande porte. A coleta de dados foi feita por aplicação de questionários estruturados com 21 questões fechadas que contemplaram dados quantitativos e qualitativos. Cada questão foi considerada como uma variável e as respostas foram analisadas descritivamente e pelo método de Análise de Correspondência. Através dos resultados pôde-se verificar que os profissionais entrevistados desconheciam o assunto, sinalizando falta de capacitação para identificar possíveis perigos, como a cisticercose. Numericamente, as empresas de porte médio foram as que apresentaram melhor nível de conhecimento sobre boas práticas de manipulação, apesar de não ter sido constatada diferença estatística relevante no nível de conhecimento sobre o complexo teniose-cisticercose em relação ao porte da empresa. Conclui-se que há necessidade de um trabalho de educação sanitária direcionado a esses profissionais.
\end{abstract}

Palavras-chave: Taenia solium, Taenia saginata, educação sanitária, vigilância sanitária, Boas Práticas de Manipulação.

\begin{abstract}
This study aimed to evaluate meat handler's knowledge of teniasis-cysticercosis complex and Good food Handling Practices in supermarkets, grocery stores, and butcher shops from Jaboticabal, São Paulo State, Brazil. For that, were evaluated 25 commercial establishments, of which nine are small merchants, ten are medium-sized shops, and six were considered as large super-markets. Data were collected with closed questionnaires with 21 questions on quantitative and qualitative information. Each question was considered as a single variable and the answers were analyzed descriptively and by correspondence analysis multivariate statistics. The data show the interviewed professionals were unaware of the issue, indicating lack of training for recognition of possible risks, as the cysticercosis. Despite the medium-sized establishments showed better results, the overall results are equivalent on the statistical analysis. Therefore, sanitary educational practices directed to these professionals are strongly recommended to improve food safety.
\end{abstract}

Keywords: Taenia solium, Taenia saginata, sanitary education, health surveillance, Good Handling Practices.

\section{Introdução}

As Boas Práticas de Manipulação (BPM) são importantes para garantir a inocuidade dos alimentos a serem adquiridos pela população. A Resolução $n^{\circ} 216$, de 15 de setembro de 2004, tem como objetivo estabelecer procedimentos de Boas Práticas para serviços de alimentação a fim de garantir condições higiênico-sanitárias adequadas para alimentos preparados e comercializados (Brasil, 2004).

Em estabelecimentos comercializadores de carne, destacase a cisticercose, a principal zoonose detectada durante o abate de bovinos (Almeida, 2006). O complexo teniose- cisticercose é constituído por duas fases distintas dos mesmos cestódeos, Taenia saginata e Taenia solium. A cisticercose bovina ocorre quando há presença da forma larval de Taenia saginata nos tecidos do hospedeiro intermediário (bovinos), enquanto a cisticercose suína ocorre quando há presença da forma larvária de Taenia solium nos tecidos do hospedeiro intermediário (suínos e, ocasionalmente, humanos) (Santos e Barros, 2009).

$\mathrm{Na}$ fase adulta habita no intestino delgado do hospedeiro definitivo, o homem, causando teniose, adquirida pelo ser humano através da ingestão de carne bovina ou suína crua ou malcozida, contendo as larvas (Santos e Barros, 2009).

\footnotetext{
${ }^{*}$ Recebido em 6 de novembro de 2013 e aceito em 30 de junho de 2014.

**Universidade Estadual Paulista UNESP, Faculdade de Ciências Agrárias e Veterinárias, Departamento de Medicina Veterinária Preventiva e Reprodução Animal, Jaboticabal, São Paulo, Brasil.

***Universidade do Estado de São Paulo, Faculdade de Zootecnia e Engenharia de Alimentos, Câmpus Pirassununga, Departamento de Medicina Veterinária, Pirassununga, São Paulo, Brasil.

Autor para correspondência: bruuh_na@hotmail.com
} 
Os hospedeiros intermediários adquirem a cisticercose ao pastar ou ingerir diretamente água contaminada, que pode ser proveniente de defecação humana contendo ovos procedentes da teniose em local inadequado, contaminando córregos e rios. O ciclo se perpetua a partir das condições precárias de higiene das instalações, tecnologia e sanidade, animais sendo alimentados com resíduos orgânicos, em convívio com seres humanos e abatidos sem inspeção veterinária (Manhoso e Prata, 2004; Santos e Barros, 2009; Oliveira et al., 2011; Pfuetzenreiter e Avila-Pires, 2000).

Objetivou-se verificar o nível de conhecimento de manipuladores de carne do município de Jaboticabal, estado de São Paulo sobre o complexo teniose-cisticercose e sobre as Boas Práticas de Manipulação, assim como também verificar se há divergência quanto a esse conhecimento entre os diferentes portes de estabelecimentos.

\section{Material e métodos}

O estudo foi desenvolvido durante o primeiro semestre do ano de 2013, no município de Jaboticabal, estado de São Paulo. Participaram do estudo manipuladores de carnes de 25 estabelecimentos, entre açougues e supermercados. Os estabelecimentos foram classificados de acordo com a quantidade média de quilos de carne comercializados em cada um, sendo considerados: de pequeno porte, quando comercializavam até cinco carcaças/semana ou $1.250 \mathrm{~kg}$ de carne semanalmente; médios, quando a quantidade vendida semanalmente estava entre 6 e 15 carcaças, ou seja, de 1.500 a $3.750 \mathrm{~kg}$; e grande, quando o valor era acima do valor para o estabelecido para os médios. A partir disso, nove foram considerados de pequeno, dez de médio e seis de grande porte.

A coleta de dados foi feita através da aplicação de um questionário estruturado com 21 questões fechadas contemplando dados quantitativos e qualitativos. Cada pergunta do questionário foi considerada como uma variável, sendo questões referentes aos temas: complexo teniose-cisticercose e Boas Práticas de Manipulação de alimentos. Em relação ao complexo, foi aferido o conhecimento acerca da epidemiologia, formas de transmissão e prevenção desta zoonose. Em relação às Boas Práticas de Manipulação, foram avaliadas as conformidades com as diretrizes preconizadas pelos órgãos regulatórios nacionais.

Foi avaliado ainda se os estabelecimentos diferem quanto ao conhecimento dos funcionários sobre os temas de acordo com o porte da empresa. As respostas dos questionários foram analisadas por estatísticas descritivas e análise multivariada, adotando-se o método de Análise de Correspondência, por meio do software Statistica ${ }^{\circledR}$, versão 7.0. AAnálise de Correspondência Multivariada pode ser classificada no conjunto de técnicas associadas a mapas perceptuais/intuitivos que facilita a aplicação e interpretação, assim como mostram a versatilidade no tratamento de variáveis. Os mapas perceptuais/intuitivos são definidos por Hairetal., 1995 como: «representação visual das percepções de objetos de um indivíduo em duas ou mais dimensões. Normalmente, este mapa tem níveis opostos de dimensões nos extremos dos eixos $\mathrm{X}$ e $\mathrm{Y}$. Cada objeto tem, então, uma posição espacial no mapa perceptual, refletindo a relativa similaridade ou preferência em relação a outros objetos segundo as dimensões do mapa perceptual.

\section{Resultados e discussão}

Dentre os 25 estabelecimentos, foram aplicados 55 questionários, sendo que destes, 13, 22 e 20 questionários foram obtidos de estabelecimentos de pequeno, médio e grande porte, respectivamente. Para melhor apresentação dos resultados, o questionário foi dividido em duas partes para a realização das análises estatísticas. As perguntas referentes a cada tema foram separadas, e aquelas referentes para ambos os temas permaneceram nos dois questionários.

Acerca do complexo teniose-cisticercose, observou-se que, apesar de $56 \%$ (31/55) dos manipuladores já terem ouvido sobre a enfermidade, o conhecimento acerca da cadeia epidemiológica é superficial, uma vez que apenas $26 \%$ (14/55) responderam que humanos são os que transmitem a doença ao animal. Em adição, 54\% (29/55) responderam que, ao ingerir carne contendo cisticerco viável, humanos desenvolvem cisticercose, e não teniose, demonstrando mais uma vez o desconhecimento a respeito da cadeia epidemiológica do complexo.

Quando questionados se a teniose é transmitida a humanos apenas pelo consumo de carne suína, $69 \%$ (38/55) responderam que não. Apesar do desconhecimento acerca de epidemiologia da doença, foi observado em todos os portes de estabelecimentos que os manipuladores sabiam que a doença traz risco à saúde humana, sendo observado no geral 95\% (52/55) de respostas "sim".

Ainda que tenha sido pequena a frequência de respostas erradas na questão "em que lugar a cisticercose é adquirida pelo animal:", ainda assim 7\% (4/55) responderam "no açougue" e $93 \%(51 / 55)$ responderam o correto "na fazenda", demonstrando que em alguns estabelecimentos os funcionários não possuem noções básicas sobre o ciclo biológico do parasito.

A maioria dos manipuladores de carne, 55\% (30/55) afirmaram não saber diferenciar o cisticerco de abscesso na carne, demonstrando que não recebem capacitação para manipulação de produtos destinados a comercialização. 58\% (32/55) dos entrevistados disseram haver formas de destruir o cisticerco da carne, sendo que $56 \%(31 / 55)$ acreditam que somente o cozimento não é suficiente para destruição do cisticerco.

Quando perguntados se há outra forma, além do cozimento, de destruir o cisticerco, 62\% (34/55) responderam que não. Entre as respostas sim, quando citaram exemplos, houve respostas como: tratamento, higiene, temperar a carne antes, 30 dias de congelamento, além de outras formas de inviabilização do cisticerco por aumento de temperatura.

Em estudo realizado por lasbik et al. (2010) para determinar a prevalência de cisticercose em suínos e de teniose em 176 propriedades localizadas na zona rural do município de Viçosa, estado de Minas Gerais, foram coletadas amostras de sangue de 226 suínos e fezes de 266 humanos. A prevalência da cisticercose suína foi de $0,4 \%$. Não foi identificada teniose humana, o que pode ser explicado por, apesar do hábito de consumir carne suína não inspecionada da própria zona rural, as pessoas se alimentavam destas bem cozidas, demonstrando a importância de conhecer formas de destruição de patógenos no alimento.

Entre os manipuladores, $76 \%$ (42/55) acreditam que uma carne procedente de frigorífico fiscalizado pelo Serviço de Inspeção 
Federal é mais segura que carnes inspecionadas por serviços estaduais ou municipais. Apesar de o valor ser considerado alto para acerto da resposta, deve-se destacar que os $24 \%$ (13/55) não reconhecem a importância da inspeção sanitária de alimentos nas indústrias, e estão sujeitos a praticarem o comércio de produtos ilegais por mero desconhecimento, o que evidencia a necessidade de treinamentos aos manipuladores no município de Jaboticabal.

Mesmo assim, 51\% (28/55) dos manipuladores afirmaram saber de algum açougue ou supermercado de Jaboticabal que vende carne obtida clandestinamente, demonstrando a necessidade de ações fiscais, além das de educação e vigilância sanitária no município.

Estatisticamente, o nível de conhecimento sobre o complexo é equivalente entre os funcionários avaliados, independentemente do porte da empresa. Isso foi verificado pela equidistância dos pontos representados pelas empresas aos pontos das respostas «sim» $\mathrm{e}$ «não». Estas correspondem à maioria das questões que compõem o questionário. Em vista dos resultados observados, nota-se um grande desconhecimento do assunto por parte dos profissionais entrevistados, possuindo pouco conhecimento sobre os riscos da ocorrência do complexo teniose-cisticercose.

Acerca do conhecimento dos manipuladores de carne sobre Boas Práticas de Manipulação de alimentos, no geral, 58\% $(32 / 55)$ destes afirmaram nunca ter participado de treinamento sobre qualidade de carne ou doenças transmitidas por alimentos. Nos estabelecimentos de pequeno porte, $62 \%$ (8/13) afirmaram não possuir manual de boas práticas disponível para os funcionários, enquanto $55 \%$ (12/22) dos médios e 70\% (14/20) dos estabelecimentos de grande porte afirmaram ter o manual no local, que após treinamentos oferecidos a esses profissionais, poderiam ser estimulados a lerem e compreenderem o manual.

Sobre a higienização do ambiente, $60 \%$ (33/55) disseram que varrem o estabelecimento a seco, o que não é recomendado nos Programas de Boas Práticas de Manipulação de Alimentos, que preconiza a lavagem com água e sabão e uso de desinfetantes, além do enxágue obrigatório para superfícies que entram em contato com alimentos. Porém, 81\% (44/55) afirmaram saber a diferença entre limpeza e desinfecção, o que facilitará o ato correto, desde que orientados a isso.

Sobre a utilização de Equipamentos de Proteção Individual, como avental, touca e botas de borracha, todos afirmaram utilizar no recinto de trabalho. Além disso, apenas $4 \%(2 / 55)$ afirmaram não lavar as mãos com água e sabonete ao iniciar, ao retomar atividades ou após manipular outros objetos. Apesar destes bons resultados no presente estudo, Oliveira et al. (2008) verificaram que $100 \%$ dos manipuladores apresentaram inadequação no item higiene das mãos (técnica) e $87,5 \%$ no que diz respeito às práticas sanitárias durante a manipulação do alimento em cozinhas de creches públicas de São Paulo por meio de um roteiro estruturado de observação com os itens considerados estritamente indispensáveis ao bom funcionamento e segurança no preparo das refeições produzidas pelas instituições. Isso leva a um fato que, apesar dos manipuladores pensarem que estão fazendo as atividades de maneira correta, podem estar praticando-as de maneira errada, evidenciando a importância do treinamento para capacitação dos manipuladores, e mostrando que o desconhecimento quanto ao assunto é comum.
Nos estabelecimentos de porte médio, somente $5 \%(1 / 21)$ afirmaram não avisar o dono do estabelecimento quando apresenta feridas na mão ou outras doenças, sendo que $25 \%$ (5/20) dos estabelecimentos grandes afirmaram não assumir esta prática. Mesmo sendo uma baixa porcentagem, é digno de preocupação, pois há chance de patógenos passarem do corpo do doente para o alimento, tornando o produto impróprio para consumo. Além disso, 67\% (36/55) dos funcionários afirmam não apresentarem atestados médicos periodicamente.

Dentre os estabelecimentos médios, 82\% (18/22) afirmaram conhecer a necessidade da lavagem semestral da caixa d'água do estabelecimento, sendo que nos estabelecimentos de pequeno porte $38 \%(5 / 13)$ e dos estabelecimentos de grande $20 \%(4 / 20)$ afirmaram não conhecer essa necessidade.

Muitos estudos têm quantificado as conformidades e conhecimento dos manipuladores de alimentos antes e após treinamentos sobre boas práticas de manipulação. Em todas podemos observar que os funcionários melhoram seus conhecimentos. Andreotti et al. (2003), a partir de um roteiro de inspeção antes e depois do treinamento, observaram que antes do treinamento, $29 \%$ dos itens estavam em conformidade em relação aos aspectos gerais de higiene pessoal de seus funcionários, e $71 \%$ inadequados. Após, $47 \%$ conformidade e $53 \%$ inadequado. O comportamento dos funcionários durante a manipulação dos alimentos também apresentou um aumento de $49 \%$ dos itens em conformidade após o treinamento, percentual de apenas $13 \%$ antes do treinamento passou para $62 \%$. Os cuidados com uniforme passaram de $38 \%$ para $75 \%$ de conformidade após o treinamento. Estes estudos demonstram a importância do treinamento e capacitação dos profissionais.

A Figura 1 mostra que as empresas de porte médio são, dentre as avaliadas, as que apresentam maior associação com as respostas "sim" do questionário. Estas correspondem às respostas esperadas no caso da avaliação de Boas Práticas de Fabricação. Justifica-se o resultado observado devido aos estabelecimentos de pequeno porte não possuírem responsáveis para capacitação dos funcionários. Já nos estabelecimentos de grande porte, pode ser observada má capacitação devido à elevada rotatividade de funcionários observada durante o estudo.

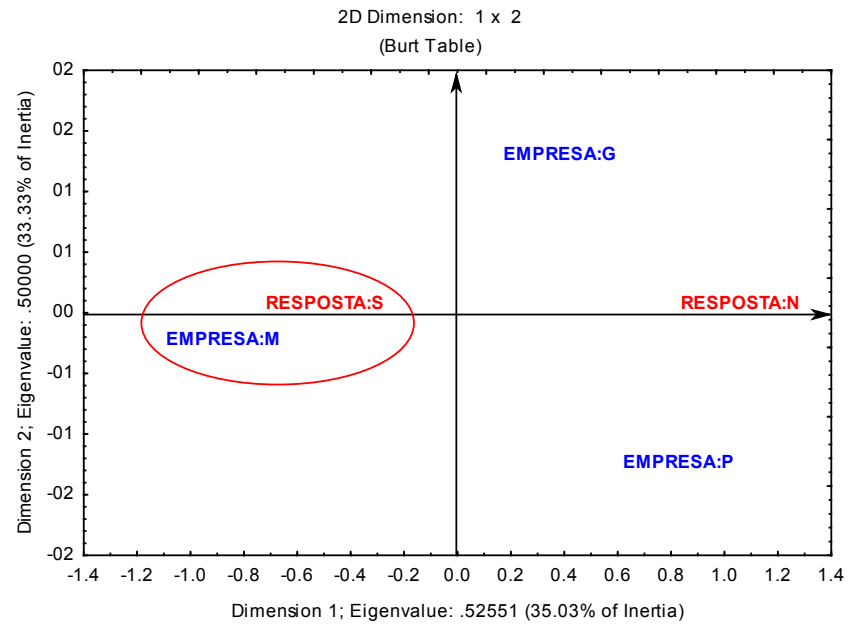

Figura 1: Mapa Perceptual do questionário sobre o conhecimento de boas práticas de manipulação de alimentos aplicado a manipuladores de carne em estabelecimentos do município de Jaboticabal, estado de São Paulo. 


\section{Conclusão}

Após verificar o desconhecimento sobre a epidemiologia do complexo teniose-cisticercose e os hábitos incorretos em relação às Boas Práticas de Manipulação por parte dos funcionários dos estabelecimentos comercializadores de carne no município de Jaboticabal-SP, conclui-se que há uma necessidade de

\section{Referências}

ALMEIDA, L. P. de; REIS, D. O.; MOREIRA, M. D.; PALMEIRA, S. B. S. Cisticercos em bovinos procedentes de Minas Gerais e abatidos em frigoríficos de Uberlândia - MG, no período de 1997 a 2001. Revista Higiene Alimentar; São Paulo, v. 20, n. 139, p. 40-43, 2006.

ANDREOTTI, A.; BELERONI, F. H.; POROSCHI, V. H. B.; PANZA, S. G. A. Importância do treinamento para manipuladores de alimentos em relação à higiene pessoal. Iniciação Científica Cesumar, v. 5, n. 1, p. 29-33, 2003.

BRASIL. Ministério da Saúde. Agência Nacional de Vigilância Sanitária. Resolução-RDC n. 216, de 15 de setembro de 2004. Dispõe sobre Regulamento Técnico de Boas Práticas para Serviços de Alimentação. Diário Oficial da União, Brasília, 2004.

HAIR, J. F.; ANDERSON, R. E; TATHAM, R. L.; BLACK, W. C. Multivariate data analysis: with readings. $4^{\text {th }} \mathrm{ed}$. Upper saddle River, New Jersey. Prentice-Hall, 1995.

IASBIK, A. F.; PINTO, P. S. de A.; BEVILACQUA, P. D.; NERO, L. A.; SANTOS, T. de O.; FELIPPE, A. G. Prevalência do complexo teníase-cisticercose na zona rural do município de Viçosa, Minas Gerais. Ciência Rural, Santa Maria, v. 40, n. 7, p. 1664-1667, 2010.

MANHOSO, F. F. R.; PRATA, L. F. Prevalência de cisticercose na região oeste do estado de São Paulo. Revista Higiene Alimentar, v. 18. n. 121. p. 42-48, 2004. implantação de programas de educação sanitária direcionada a esses profissionais no município, papel este que deve ser assumido pela vigilância sanitária em maior contribuição à educação dos profissionais do que simplesmente a fiscalização do local. Desta forma, é necessária uma mudança de comportamento e de atitudes, o que contribuirá para a redução da presença de riscos nos alimentos ofertados à população nesse município.

OLIVEIRA, A. W. de; OLIVEIRA, J. A. C. de; BATISTA, T. G.; OLIVEIRA, E. R. A. de; CAVALCANTI NETO, C. C.; ESPÍNDOLA FILHO, A. M.. Estudo da prevalência da cisticercose bovina no estado de Alagoas.Acta Veterinária Brasílica, v. 5, n. 1, p. 41-46, 2011.

OLIVEIRA, M. N.; BRASIL, A. L. D.; TADDEI, J.A. A. C. Avaliação das condições higiênico-sanitárias das cozinhas de creches públicas e filantrópicas. Ciência e Saúde Coletiva. v.13, n. 3. p. 1051-1060, 2008.

OLIVEIRA, R. B. A.; ROLIM, M. B. Q.; MOURA, A. P. B. L.; MOTA, R. A. Avaliação higiênico-sanitária dos boxes que comercializam carnes em dois mercados públicos da cidade do Recife-PE/Brasil. Medicina Veterinária, v. 2, n. 4, p. 10-16, 2008. Disponível em: http://www.revista.dmv.ufrpe.br/revista/index.php/rdmv/article/ view/67/67. Acesso em14 out. 2013.

PFEUTZENREITER, M. R; ÁVILA-PIRES, F. D de. Epidemiologia da teníase/cisticercose por Taenia solium e Taenia saginata.

Ciência Rural, v. 30, n. 3, p. 541-548, 2000.

SANTOS, J. M.. G dos; BARROS, M. C. R. B. Cysticercus bovis e Cysticercus cellulosae: endoparasitas de importância no comércio da carne. Revista em Agronegócios e Meio Ambiente, v. 2, n. 1, p. 21-39, 2009. 